\title{
Hepatitis C coinfection adversely affects the life expectancy of people living with HIV in northwestern Poland
}

\author{
Magdalena Leszczyszyn-Pynka ${ }^{1}$, Piotr Ciejak ${ }^{1}$, Katarzyna Maciejewska ${ }^{1}$, \\ Magdalena Witak-Jędraํㄹ Malwina Karasińska-Cieślak², Ewa Karpińska³, \\ Marta Wawrzynowicz-Syczewska ${ }^{3}$, Miłosz Parczewski ${ }^{1}$
}

\author{
${ }^{1}$ Department of Infectious, Tropical Diseases and Immune Deficiency, \\ Pomeranian Medical University, Szczecin, Poland \\ 2Department of Infectious, Tropical Diseases and Immune Deficiency, \\ Regional Hospital, Szczecin, Poland \\ 3Department of Infectious Diseases, Hepatology and Liver Transplantation, \\ Pomeranian Medical University, Szczecin, Poland
}

Submitted: 3 December 2015

Accepted: 1 February 2016

Arch Med Sci 2018; 14, 3: 554-559

DOI: https://doi.org/10.5114/aoms.2016.58744

Copyright () 2016 Termedia \& Banach

\section{Abstract}

Introduction: Hepatitis C (HCV) infection adversely affects survival among people living with HIV, increasing mortality risk due to liver-related causes. In Poland HCV is found among $\sim 30 \%$ of HIV infected individuals, with only a small percentage successfully treated for this coinfection. This study aimed to analyze the HCV-associated influence on the life expectancy among HIV/HCV coinfected patients from northwestern Poland.

Material and methods: Longitudinal data of 701 (368 HIV monoinfected and $368 \mathrm{HIV} / \mathrm{HCV}$ coinfected) patients were investigated to assess the life expectancy and survival after HIV diagnosis. Kaplan-Meier and Cox analyses were used to assess the mortality risk in both unadjusted and multivariate models. Effect plots indicate the adjusted hazard ratio for HCV-associated survival. Results: Overall mortality was significantly higher among HCV coinfected (22.52\%) compared to HIV monoinfected $(10.32 \%)$ cases $(p<0.001$, OR $=2.52(95 \% \mathrm{Cl}: 1.65-3.85))$, with shorter life expectancy among HIV/HCV infected patients (median: 55.4 (IQR: 42.8-59.1) years) compared to HIV monoinfection (median 72.7 (IQR: 60.4-76.8) years, univariate $H R=4.15$ (95\% Cl: 2.7-6.38), $p<0.0001$, adjusted $\mathrm{HR}=2.32(95 \% \mathrm{Cl}: 1.47-3.65)$, $p<0.0001)$. After HIV diagnosis, HCV adversely influenced the survival after 15 years of follow-up, with a strengthened impact in the subsequent 5 years (univariate $\mathrm{HR}=1.57(95 \% \mathrm{Cl}: 1.05-2.34) p=0.026$ for the 20 -year survival time point, adjusted $\mathrm{HR}=2.21$ (95\% Cl: 1.18-4.13), $p=0.013$ ).

Conclusions: Among patients living with HIV, HCV coinfection is associated with a median life expectancy decrease of 17.3 years and low probability of surviving until the age of 65 years. In the era of directly acting anti-HCV drugs, treatment scale-up and immediacy of treatment are advisable in this cohort.

Key words: HIV/HCV coinfection, mortality, hepatitis C virus treatment.

\section{Introduction}

Due to shared routes of transmission, hepatitis $C$ coinfection is found in approximately one-third of the people living with HIV. In Europe sig-

\section{Corresponding author:} Milosz Parczewski MD, PhD Department of Infectious, Tropical Diseases and Immune Deficiency Pomeranian Medical University

4 Arkońska St

71-455 Szczecin, Poland Phone: +48 918139456 Fax: +48 918139449 E-mail: mparczewski@yahoo.co.uk 
nificant differences in the prevalence are noted. Rates of Hepatitis C virus (HCV) coinfection range from $50 \%$ in the eastern regions where injection drug use (IDU) is fuelling the epidemics, $30 \%$ in the central and southern regions to $<20 \%$ in the northern areas [1, 2]. Recently observed outbreaks of acute HCV among men who have sex with men (MSM) also increase the number of coinfected patients [3]. In the era of effective antiretroviral therapy, among individuals with immune recovery HIV infection does not adversely affect the life expectancy [4]. It should be noted, however, that survival among HCV coinfected individuals is notably shorter compared to HIV monoinfected cases. In this group a notable decrease in the survival rates and increased mortality have been consistently reported across Europe and worldwide [5, 6]. Persons living with HIV/HCV are at a higher risk of liver-related death, hepatocellular carcinoma (HCC) or hepatic decompensation [7]. Data from the EuroSIDA and D:A:D studies indicate that among anti-HCV positive individuals living with HIV, liver-related events are the second, after AIDS, cause of mortality, accounting for $21.6 \%$ of all deaths. Mortality was the highest in patients aged 35-45 with liver tissue fibrosis $\geq F 2$ and lymphocyte $T$ CD4 counts $<300$ cells/ $\mu$ l [8].

In Poland, the HIV epidemic has been historically associated with injection drug use, with an outbreak in the years 1989-1990 and a subsequent shift to predominance of sexual transmissions from 2007 [9]. Preventive strategies, such as needle exchange as well as methadone programs, coupled with economic growth, resulted in a reduction in the number of new HIV/HCV cases; however, hepatitis $C$ replication remains active in approximately $30 \%$ of individuals living with HIV [10]. So far, uptake of HCV treatment has been at the level of $\sim 15 \%$ of the total number of diagnosed cases for both HCV monoinfected [11] and HIV/HCV coinfected patients [12]. There are only incidental reports on the efficacy of anti-HCV treatment in the Polish HIV infected population, with a sustained virologic response achieved in $<40 \%$ of treated cases for the treatments based on pegylated interferon and ribavarin [13]. There are no data on life expectancy of HIV/HCV coinfected patients. Currently, HCV treatment is regulated by national guidelines with no priority for HIV patients. Our study aimed to bridge the gap in this knowledge and to investigate the $\mathrm{HCV}$ associated influence on overall life expectancy as well as survival after HIV diagnosis.

\section{Material and methods}

For the study longitudinal data of 701 patients with known HCV status followed up from January 1996 until the end of December 2014 at the Department of Infectious, Tropical Diseases and Im- mune Deficiency, Pomeranian Medical University, Szczecin, Poland and the Outpatient Clinic for Acquired Immunodeficiency, Regional Hospital, Szczecin, Poland were included. The study protocol was approved by the bioethical committee of Pomeranian Medical University, Szczecin, Poland (approval number BN-001/34/04). Life expectancy was calculated using the date of birth, duration of the HIV infection from the date of the first positive HIV screening test if later confirmed by Western blot, immunoblotting or serum HIV RNA. The following data were collected: age, gender, route of transmission, hepatitis C co-infection serology, HCV-RNA, $\mathrm{HCV}$ genotype, clinical category at diagnosis according to CDC 1993 case definition, date of death, baseline HIV viral load, and baseline T CD4+ lymphocyte count. Baseline T CD4+ lymphocyte count was defined as the first documented result after the HIV diagnosis. CDC category was assumed based on a review of the clinical record of the patient. In cases of late care entry category A (asymptomatic stage of HIV infection) was assumed if no apparent immunodeficiency was reported or available from medical records. Data on chronic hepatitis B were not included in the analysis due to the small number of confirmed HIV/HBV co-infection cases. The study endpoint was defined as all-cause mortality excluding cases with documented accidental death. The end of observation date (study endpoint) was defined as either the death date, last recorded date of visit (cases lost to follow-up) or $31^{\text {st }}$ of December 2014 for the patients remaining under care (termination of data collection). Whenever it was impossible to determine the exact date of death ( 6 cases) the median date between the last recorded visit and information of death was assumed as the death date.

\section{Statistical analysis}

Statistical analyses for nominal variables were performed with the $\chi^{2}$ test (gender, HIV infection stage at genotyping, transmission route), while for continuous variables (age at HIV diagnosis, T CD4 ${ }^{+}$ lymphocyte count, HIV viral load at care entry) the Mann-Whitney U-test was used (Statistica v. 12 software, StatSoft, Poland). Kaplan-Meier cumulative mortality was calculated, and statistical significance of survival data was analyzed using a log-rank test. Unadjusted and multivariate Cox proportional hazards models were used to assess the effect of analyzed parameters on the risk of death and to calculate the hazard ratios (HR). $P$-values of 0.05 were considered significant.

\section{Results}

Among 701 analyzed patients, 333 (47.5\%) cases were HCV positive and $368 \mathrm{HCV}$ (52.5\%) 
M. Leszczyszyn-Pynka, P. Ciejak, K. Maciejewska, M. Witak-Jędra, M. Karasińska-Cieślak, E. Karpińska, M. Wawrzynowicz-Syczewska, M. Parczewski

Table I. Basic characteristics of HCV/HCV vs. HIV monoinfected patients

\begin{tabular}{|c|c|c|c|c|}
\hline Parameter & $\begin{array}{l}\text { HIV mono- } \\
\text { infected }\end{array}$ & $\begin{array}{l}\text { HIV/HCV } \\
\text { coinfected }\end{array}$ & $P$-value & Total \\
\hline Male, $n(\%)$ & $262(71.2)$ & $247(74.2)$ & 0.37 & $509(72.6)$ \\
\hline Age at HIV diagnosis, median (IQR) [years] & $34.0(27.1-43.4)$ & $28.1(23.7-34.1)$ & $<0.001$ & $30.9(25.5-38.3)$ \\
\hline \multicolumn{5}{|l|}{ HIV infection stage at genotyping, $n(\%)^{*}$ : } \\
\hline A & $122(38.1)$ & $136(45.5)$ & \multirow[t]{3}{*}{$<0.001$} & $258(41.7)$ \\
\hline B & $102(31.9)$ & $113(37.8)$ & & $215(34.7)$ \\
\hline C & $96(30.0)$ & $50(16.7)$ & & $146(23.6)$ \\
\hline \multicolumn{5}{|l|}{ Dominant transmission route, $n\left(\%\right.$ of total) ${ }^{\star}$ : } \\
\hline IDU (intravenous drug use) & $22(6.8)$ & $257(83.7)$ & \multirow[t]{3}{*}{$<0.001$} & $279(44.2)$ \\
\hline MSM (men having sex with men) & $143(44.0)$ & $19(6.2)$ & & $162(25.6)$ \\
\hline HET (heterosexual) & $160(49.2)$ & $31(10.1)$ & & $191(30.2)$ \\
\hline $\begin{array}{l}\text { Lymphocyte CD4+ } T \text { cell count at care entry, } \\
\text { median (IQR) }\end{array}$ & $250(70-519)$ & $283(116-475)$ & 0.169 & $277(93-496)$ \\
\hline $\begin{array}{l}\text { HIV viral load at care entry, } \\
\text { mean log copies/ml (SD) }\end{array}$ & $4.98(4.31-5.61)$ & $\begin{array}{c}4.15 \\
(4.72-5.2)\end{array}$ & 0.003 & $4.91(4.21-5.52)$ \\
\hline
\end{tabular}

${ }^{\star} C D C$ category not available for 82 individuals, transmission route not available for 69 cases.

negative. HCV coinfected patients were younger at the time of HIV diagnosis than HIV monoinfected individuals (28 (IQR: 23.7-34.1) vs. 34.0 (27.143.4) years, $p<0.001)$, less likely diagnosed with AIDS (16.7\% vs. $30 \%, p<0.001)$, and presented with a lower HIV-1 viral load at care entry (4.15 (IQR: 4.72-5.2) vs. 4.98 (4.31-5.61) log copies/ml). Injection drug use was the dominant transmission route in this group (83.7\%) (Table I). HCV genotype distributions (available for 152 coinfected individuals) were as follows: genotype 1: 73 (48.34\%) cases (1a: 13 (8.55\%) 1b: 57 (37.5\%), with unde-

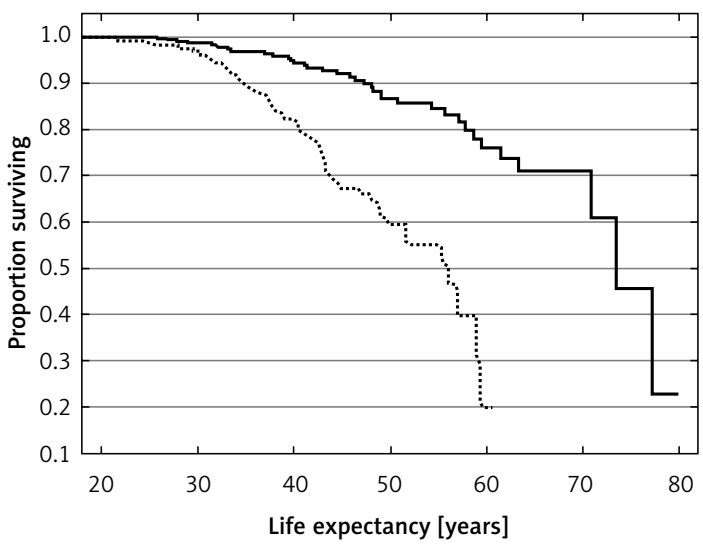

Group sizes:

HIV $\quad \begin{array}{lllllllllll}368 & 344 & 283 & 186 & 130 & 77 & 38 & 14 & 4 & 1\end{array}$

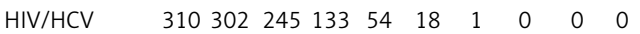

— HIV monoinfected $\quad$....... HIV/HCV coinfected Log-rank $p<0.0001$

Figure 1. Kaplan-Meier plots for probability of longterm survival among HIV monoinfected and HIV/ HCV coinfected patients termined subgenotype: $3(1.97 \%))$, genotype $3 a$ : 45 (29.8\%) patients, genotype 4: 33 (21.7\%), individuals and genotype $3 / 4$ found in $1(0.65 \%)$ case. It should be noted that there were 53 patients treated with pegylated interferon-ribavirin for the hepatitis C infection. Of these, only 21 (39.6\%) achieved a sustained virological response (SVR). All patients with HCV treatment success remain alive. Patients with sustained virological response (SVR) were excluded from the survival analyses.

Cumulative observation time from birth was 28876.75 person-years with 5731.4 person-years in the period from HIV diagnosis until the end of the study. In the analyzed cohort 113 (16.1\%) deaths were recorded, with overall mortality rates notably higher among HCV coinfected cases $(n=$ 75, 22.52\%) compared to HIV monoinfected ones $(n=38,10.32 \%)(p<0.001, \mathrm{OR}=2.52(95 \% \mathrm{Cl}$ : 1.65-3.85)).

Median overall Kaplan-Meier estimated life expectancy was 69.6 (IQR: 50.87-75.79) years, being notably shorter among HIV/HCV infected patients (median: 55.4 (IQR: 42.8-59.1) years) than among HIV monoinfected ones (median 72.7 (IQR: 60.476.8 ) years). HCV coinfection was associated with decreased life expectancy (log rank $p<0.0001$, univariate $\mathrm{HR}=4.15$ (95\% Cl: 2.7-6.38) for the Cox model, $p<0.0001$ ); strikingly, none of these patients reached the age of 65 years (Figure 1). After adjustment for age and gender - variables significantly associated with differences in life expectancy in the analyzed cohort - the negative influence of HCV infection on the life expectancy remained significant $(\mathrm{HR}=2.32(95 \% \mathrm{Cl}: 1.47-3.65))$, $p<0.0001$. 
In the survival analysis using the HIV diagnosis as the initial time point, HCV infection did not influence the modeled survival for the first 14 years of the follow-up, reaching statistical significance after 15 years of observation (log-rank $p=0.0462$, univariate $H R=1.506(95 \% \mathrm{Cl}: 1.01-2.25)$ for the Cox model, $p=0.0458)$. This impact was strengthened in the subsequent years (log-rank $p$-value $=$ 0.026 for the time point of 20-year survival with HIV diagnosis; univariate $\mathrm{HR}=1.57$ (95\% Cl: 1.052.34) for the Cox model, $p=0.026$ ) (Figure $2 \mathrm{~A}$ ). In the multivariate analysis of the 20-year survival with HIV diagnosis, after adjustment for the HIV-associated variables (HIV viral load > 100000 copies/ml, T CD4+ lymphocyte count < 200 copies/ $\mu \mathrm{l})$, gender and age, HCV coinfection proved to be independently associated with higher likelihood of death $(\mathrm{HR}=2.21$ (95\% Cl: 1.18-4.13), $p=0.013)$ (Figure $2 \mathrm{~B}$ ). The power of this association was lost after adjustment for the history of drug use $(\mathrm{HR}=0.67$ (95\% Cl: 0.28-1.64), $p=0.038)$. There were no differences in survival probability associated with HCV genotypes and HCV viremia; however, these data were available only for 151 cases.

\section{Discussion}

Data from this study confirm that hepatitis C coinfection is an independent factor negatively influencing survival among HIV infected patients. Importantly, the deleterious effect of HCV was found in the population younger at HIV diagnosis, with higher frequency of asymptomatic infections and a lower number of AIDS cases at baseline as well as a lower burden of HIV replication. The HIV monoinfected and HIV/HCV infected groups differed significantly by age at diagnosis, infection stage, transmission route of infection and the HIV viral load at baseline. Patients coinfected with hepatitis $C$ were notably younger, more likely asymptomatic with a lower HIV viral load compared to monoinfected cases, which may be associated with earlier diagnoses among these subjects. It should be noted that the monoinfected group includes both MSM and a significant number of heterosexually infected women, known to be diagnosed at a later age with more advanced disease and higher HIV viremia [14]. A similar phenomenon was observed previously for the nationwide datasets [15]. Differences in the transmission route were associated with the increased risk of hepatitis $C$ acquisition among injection drug users and reflect the distribution of this coinfection among HIV infected cohorts in Europe [1, 16].

We have previously reported partial data on this cohort calculated for 507 individuals, with liver-related mortality accounting for $21 \%$ of non-AIDS related deaths [17]; this dataset has been expanded to 701 cases in the current study and included only patients with available HCV serology. Coinfection with hepatitis $C$ negatively influenced the life expectancy, with no patient surviving until the age of 65 years. A similar finding has been reported in the
A

$\begin{array}{lcccc}\text { Survival period } & 60 & 120 & 180 & 240 \\ \text { [months]: } & & & & \\ \text { Log-rank } p \text {-value: } & 0.92 & 0.18 & 0.046 & 0.026\end{array}$

B

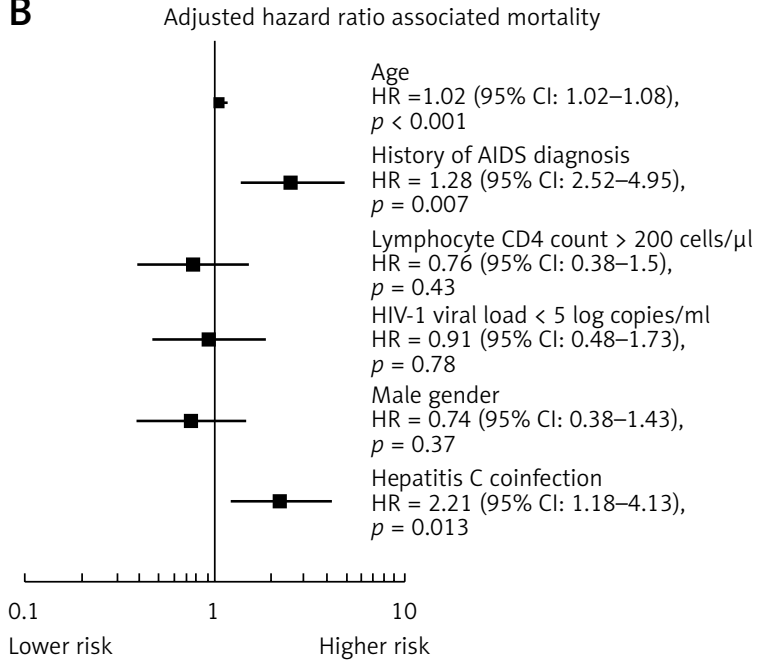

Group sizes:

HIV $\quad \begin{array}{llllllllllll}368 & 283 & 224 & 172 & 135 & 109 & 95 & 68 & 55 & 39 & 22 & 9\end{array}$

HIV/HCV $310283258228199169139113 \quad 92 \quad 64 \quad 38 \quad 23$

- HIV monoinfected $\quad$....... HIV/HCV coinfected

Log-rank $p<0.0001$

Figure 2. A - Kaplan-Meier plots indicating probability of survival after HIV diagnosis among HIV monoinfected and HIV/HCV coinfected patients. B - Multivariate Cox regression plots presenting adjusted hazard ratios associated with mortality at the time point of 20 years follow-up with HIV. Squares represent adjusted hazard ratio (HR) for the parameters, lines represent confidence intervals for the HR 
USA, with only $6 \%$ of coinfected individuals reaching this age threshold and the median age of death being 52 (95\% Cl: 46.8-57.3) years [18]. After HIV diagnosis the negative impact on survival was not immediate, with 15 years necessary for HCV to exert a significant effect on mortality. This influence was independent of T CD4+ lymphocyte count, AIDS diagnosis and age. This finding is similar to the data presented by Grint et al., with increased likelihood of death related to age, $\mathrm{T} C D 4^{+}$lymphocyte cell count, history of antiretroviral drug use, HBV coinfection, duration of HCV infection and liver fibrosis [19]. Additionally, in our study the effect on mortality was not related to the HCV genotype or viral load, which is in line with the previously published data [20]. In D:A:D cohorts, in the years 1999-2011 liver-related mortality accounted for $13 \%$ of deaths, being the second, after neoplastic disease, most common cause of non-AIDS related deaths. It should also be noted that hepatic mortality was almost invariably (95\%) associated with HCV or HBV infection [21]. After low T CD4+ lymphocyte count, associated with a 16.1-fold (T CD4+ lymphocyte count $<50$ cells $/ \mu \mathrm{l}$ ) increase in mortality risk, HCV coinfection was the second key factor decreasing survival (the hazard ratio increased 6.66-fold compared to monoinfected subjects) [8]. This effect might be related to the faster progression of liver fibrosis.

It should also be emphasized that in our cohort HIV/HCV individuals were mostly (83.7\%) injection drug users. This population is vulnerable not only to the effects of substance abuse and higher liver-related mortality. Also, the risk of AIDS-related death and mortality due to other causes has been accentuated in these individuals [22]. As a result, IDUs do not benefit from the higher life expectancy observed among antiretroviral treated people living with HIV [4].

Anti-HCV treatment may increase the life expectancy among individuals achieving a SVR. Indeed, in our study none of the patients with SVR died, although this may be due to the selection of patients prior to the introduction of treatment with pegylated interferon/ribavirin. A survival benefit was reported by Berenguer et al. [23] for interferon-based treatment, but the effect of treatment with directly acting HCV antivirals on survival is yet to be established.

The study has the following limitations: Firstly, the HCV-related mortality in the HIV/HCV coinfected group is confounded by the overall higher mortality risk among injection drug users - the dominant group with HIV/HCV coinfection. However, liver-related deaths including complications of hepatitis $C$ remain a key mortality issue even in this group [24], as discussed above. Also, survival analyses are typically censored if the exact date of death is unknown. To overcome this limitation, in 6 cases with the missing death time point, the median date between the last observation time and information that the patients actually died was used.

In conclusion, our study indicates the deleterious effect of HCV coinfection on long-term survival after HIV diagnosis and models a median life expectancy decrease of 17.3 years among $\mathrm{HIV} / \mathrm{HCV}$ coinfected subjects. Additionally, HCV coinfected patients do not survive until old age, currently defined by the WHO at the threshold of 65 years. In the era of directly acting anti-HCV drugs, scale-up and immediacy of treatment, as stated in EASL guidelines, are advisable in this cohort [25].

\section{Acknowledgments}

The study was financially supported by a grant from the Polish Ministry of Science, grant number NCN NN 402613840, and internal funds from the Pomeranian Medical University.

\section{Conflict of interest}

The authors declare no conflict of interest.

\section{References}

1. Peters L, Mocroft A, Lundgren J, Grint D, Kirk O, Rockstroh J. HIV and hepatitis C co-infection in Europe, Israel and Argentina: a EuroSIDA perspective. BMC Infect Dis 2014; 14 Suppl. 6: S13.

2. Grzeszczuk A, Wandalowicz AD, Jaroszewicz J, Flisiak R. Prevalence and risk factors of HCV/HIV co-infection and HCV genotype distribution in North-Eastern Poland. Hepat Mon 2015; 15: e27740.

3. Kaplan-Lewis E, Fierer DS. Acute HCV in HIV-infected MSM: modes of acquisition, liver fibrosis, and treatment. Curr HIV/AIDS Rep 2015; 12: 317-25.

4. Lewden C, Bouteloup V, De Wit S, et al. All-cause mortality in treated HIV-infected adults with CD4 $>/=500 /$ $\mathrm{mm} 3$ compared with the general population: evidence from a large European observational cohort collaboration. Int J Epidemiol 2012; 41: 433-45.

5. Hernando V, Perez-Cachafeiro S, Lewden C, et al. Allcause and liver-related mortality in HIV positive subjects compared to the general population: differences by HCV co-infection. J Hepatol 2012; 57: 743-51.

6. Klein MB, Rollet-Kurhajec KC, Moodie EE, et al. Mortality in HIV-hepatitis C co-infected patients in Canada compared to the general Canadian population (2003-2013). AIDS 2014; 28: 1957-65.

7. Morlat P, Roussillon C, Henard S, et al. Causes of death among HIV-infected patients in France in 2010 (national survey): trends since 2000. AIDS 2014; 28: 1181-91.

8. Weber R, Sabin CA, Friis-Moller N, et al. Liver-related deaths in persons infected with the human immunodeficiency virus: the D:A:D study. Arch Intern Med 2006; 166: 1632-41.

9. Rosinska M, Zielinski A. Recent increase in HIV rate by age, cohort, period analysis of surveillance data suggests changes in HIV epidemiology in Poland. Cent Eur J Public Health 2011; 19: 123-7. 
10. Niedzwiedzka-Stadnik M, Pielacha M, Rosinska M. HIV and AIDS in Poland in 2012. Przegl Epidemiol 2014; 68: 283-9, 383-6.

11. Flisiak R, Halota W, Tomasiewicz K, Kostrzewska K, Razavi HA, Gower EE. Forecasting the disease burden of chronic hepatitis C virus in Poland. Eur J Gastroenterol Hepatol 2015; 27: 70-6.

12. Cielniak ISE, Weber-Kaniuk I, Firląg-Burkacka E, Horban A. Predictors of response to chronic hepatitis C therapy in HIV positive patients - data from outpatient clinic in Warsaw, Poland. 14 $4^{\text {th }}$ European Clinical Society Conference, October 19-19, Brussels, Belgium. Abstract PE13/23. 2014.

13. Jablonowska E, Piekarska A, Koslinska-Berkan E, Omulecka A, Szymanska B, Wojcik K. Sustained virologic response and IL28B single-nucleotide polymorphisms in patients with chronic hepatitis $C$ treated with pegylated interferon alfa and ribavirin. Acta Biochim Pol 2012; 59: 333-7.

14. Parczewski M, Leszczyszyn-Pynka M, Bander D, Urbanska A, Stanczak G, Boron-Kaczmarska A. Characteristics of HIV-1 non-B subtype infections in Northwest Poland. J Med Virol 2010; 82: 1306-13.

15. Parczewski $M$, Leszczyszyn-Pynka $M$, Witak-Jedra $M$, et al. Transmitted HIV drug resistance in antiretroviraltreatment-naive patients from Poland differs by transmission category and subtype. J Antimicrob Chemother 2015; 70: 233-42.

16. Peters L, Klein MB. Epidemiology of hepatitis C virus in HIV-infected patients. Curr Opin HIV AIDS 2015; 10: 297-302.

17. Parczewski $M$, Bander D, Leszczyszyn-Pynka $M$, et al. Risk of all-cause mortality in HIV infected patients is associated with clinical, immunologic predictors and the CCR5 Delta32 deletion. PLoS One 2011; 6: e22215.

18. Pinchoff J, Drobnik A, Bornschlegel K, et al. Deaths among people with hepatitis C in New York City, 20002011. Clin Infect Dis 2014; 58: 1047-54.

19. Grint DPL, Kovari H, Rockstroh J, Lacombe K. EuroSIDA and the Swiss HIV Cohort Study in EuroCoord. A Validated Prognostic Score Estimating the Risk of Liver-Related Death Among HIV/HCV Coinfected Individuals. Abstract 637, 22nd Conference on Retroviruses and Opportunistic Infections. 2015.

20. Rockstroh JK, Peters L, Grint D, et al. Does hepatitis C viremia or genotype predict the risk of mortality in individuals co-infected with HIV? J Hepatol 2013; 59: 213-20.

21. Smith CJ, Ryom L, Weber R, et al. Trends in underlying causes of death in people with HIV from 1999 to 2011 (D:A:D): a multicohort collaboration. Lancet 2014; 384: 241-8.

22. Murray M, Hogg RS, Lima VD, et al. The effect of injecting drug use history on disease progression and death among HIV-positive individuals initiating combination antiretroviral therapy: collaborative cohort analysis. HIV Med 2012; 13: 89-97.

23. Berenguer J, Rodriguez E, Miralles P, et al. Sustained virological response to interferon plus ribavirin reduces non-liver-related mortality in patients coinfected with HIV and hepatitis C virus. Clin Infect Dis 2012; 55: 728-36.

24. Grint D, Peters L, Rockstroh JK, et al. Liver-related death among HIV/hepatitis C virus-co-infected individuals: implications for the era of directly acting antivirals. AIDS 2015; 29: 1205-15.

25. EASL Recommendations on treatment of hepatitis $C$ 2015. J Hepatol 2015; 63: 199-236. 